\title{
44. COMMISSION DES OBSERVATIONS ASTRONOMIQUES AU-DEHORS DE L'ATMOSPHERE TERRESTRE
}

\section{Report of Meetings}

President : L. Goldberg.

SeCretary: Miss E. A. Müller.

\section{Administrative Meeting, 27 August 1964}

During this session, only business matters were discussed.

r. The Commission approved the proposed composition of the Organizing Committee:

President: L. Goldberg.

Vice-President: Mrs A. G. Massevitch.

Members of the Organizing Committee: A. Dollfus, R. Lüst, F. G. Smith, P. Swings, H. C. van de Hulst.

2. For the general information of the Commission the President read resolutions (4) and (5) which were adopted by the Inter-Union Commission on the Ionosphere at its meeting in Tokyo, September 1963 . The following are the resolutions in question:

Resolution (4): 'Recognizing the great value of measurements of solar X-ray and extreme UV ionizing radiation flux for understanding and interpreting the ionosphere, we recommend that continuous recordings on certain wavelengths be given high priority in artificial satellite instrumentation. Emphasis should be placed on a co-ordinated sequence of relatively simple measurements, and on the continuity. It is of special importance to cover the period of the IQSY.' The President mentioned that resolution (4) is being implemented.

Resolution (5): 'The well-standardized daily measurements of solar emission at $10.7 \mathrm{~cm}$ and neighboring wavelengths have greatly assisted our endeavors to understand the solar UV and $\mathrm{X}$-ray emissions which ionize and heat the Earth's upper atmosphere.

It is recommended that the making of precision routine measurements be encouraged at wavelengths between I and $25 \mathrm{~cm}$. It is also recommended that in this wavelength range there should be continuous runs of well resolved spectroheliograms which will permit discrimination between the various active areas and the background.'

3. In response to a request from the General Secretary, the President asked the members present to consider in general how the future work of the Commission should be organized and, specifically, whether the Commission should become a technical committee that would be concerned only with the techniques of space astronomy. It was the unanimous opinion of all members taking part in the discussion that, at least at the present stage of the development of space astronomy, space techniques should not be isolated from the relevant subject matter. P. Swings considers the present structure of the Commission to be most valuable because it provides a link between all astronomical topics studied from space vehicles. He pointed out that the President's Draft Report demonstrated that there was no serious duplication between the work of Commission 44 and that of Commissions dealing with related subject matter. R. Lüst emphasized that membership in the Commission could be used as a mechanism for bringing physicists into the IAU. As an example, he pointed out that gamma ray astronomy is not within the province of any other one Commission and must remain as a responsibility of Commission 44 . 
4. P. Swings congratulated the President for having prepared such an excellent Draft Report in which the many different aspects and topics of space research were brought together. Members of the Commission were requested to inform the President of any needed corrections prior to publication of the Report in the Transactions.

5. With respect to future scientific meetings that might be sponsored by the Commission, the President pointed out that symposia such as that held recently at Liège, while extremely useful as a form for the presentation of scientific results, are too large to permit fruitful discussion of many subjects. He suggested an increase in the number of smaller meetings of a more specialized character between General Assemblies.

$P$. Swings suggested that one colloquium each year with a small number of participants might be useful, and that another large symposium might be held in 1967. Miss Roman referred to a need for small meetings dealing with the techniques of space astronomy. $D$. Walsh felt that it was very important to hold small meetings dealing with space radio astronomy, in view of the very specialized nature of the subject. The President offered the help of the Organizing Committee in arranging such small meetings when desired.

\section{Scientific Meeting, 28 August 1964}

This scientific session was devoted primarily to a survey of future space research experiments planned in different countries. The President remarked that IAU Symposium no. 23, held in Liège a week earlier, was concerned only with new results obtained from space vehicles and specifically excluded plans for new experiments.

The session began with a series of invited papers as follows: (I) 'General Problems of ESRO' (R. Lüst); (2) 'The Sun' (C. de Jager, H. J. Smith, V. K. Prokofiev); (3) 'Moon, Planets, Comets, and Interplanetary Medium' (L. Biermann, U. Liddel, V. K. Prokofiev); (4) 'Stars and Stellar Systems' (R. Lüst, P. Swings, Nancy Roman, V. K. Prokofiev). The invited program was followed by several short communications given by Y. Fujita, J. Houtgast, G. Newkirk, Z. Suemoto, G. Elwert, and N. Boneff.

INVITED PROGRAM

\section{(I) R. Lüst. Scientific aims of ESRO}

Very preliminary plans for the planned European Space Research Organization (ESRO) were reported at the last meeting of the IAU in Berkeley. After 3 years of preparation by the so-called Preparatory Commission, this new European Organization formally came into existence on 20 March 1964.

Since some of the astronomical projects of this organization will be discussed during this session and since the organization is so new, it might be helpful to discuss very briefly the general scientific aims of ESRO. The plans proposed for the organization are a direct result of its scientific program as stated in the Convention: 'The purpose of the organization shall be to provide for and to promote collaboration among European states in space research and technology exclusively for peaceful purposes.'

The following are some of the boundary conditions, which served as a guide and which should be kept in mind in discussing the planned program:

I. The scientific aims (which have emerged as a result of the proposals from scientific groups in the member states).

2. The technological capabilities in Europe.

3. The accomplishments and plans in space research in other parts of the world.

4. The financial ceiling of an eight year period sets the limit to the scientific work. 
It is, of course, clear that it will not be possible to set up a detailed scientific program as much as 8 years in advance. On the other hand it is necessary to envisage a possible program in order to plan facilities required and to satisfy the member states that a useful scientific program can be achieved within the agreed financial limit. In addition, certain projects come to fruition in $2 \frac{1}{2}$ to 5 years' time and, therefore, some parts of the scientific program must be decided upon early, at least in broad outline.

In outlining the scientific aims of ESRO it must be made clear that most of the scientific work (planning of experiments, design and construction of scientific measuring instruments, interpretation and publication of scientific results) will be carried out by research groups in the member states, e.g. in existing astronomical observatories. The cost of these activities will be borne by national funds. Only in major programs, such as those associated with a large astronomical satellite, will the main scientific instruments be developed, constructed and financed under the control of ESRO.

For the smaller projects, the task and the responsibility of ESRO will be to integrate the experiments from the different scientific groups into space vehicles, to provide, launch and track the vehicles and finally to recover the experimental data.

The scientific program consists of rocket and satellite experiments. So far, some 70 experiments have been proposed for rockets and about the same number for satellites or space probes. Of these, about 25 are in astrophysics, including solar physics, in studies of planets and comets, and in general stellar astronomy.

The first two sounding rockets were launched 6 weeks ago from Sardinia. Both rockets contained release experiments, by the groups at Liège and Munich. The aims of these experiments were the observation of ammonia and barium clouds, connected with studies of the physics of comets and comet tails. The experiments were successful.

More sounding rocket launchings are planned for this year from Sardinia and Ile du Levant with the British Skylark and the French Centaure rockets. Beginning with the second year it is hoped to launch 40 sounding rockets of which about 60 per cent will be Centaure rockets and 40 per cent Skylarks. If, however, the complexity and correspondingly the cost of the rocket increases, as for example by the introduction of stabilization, the numbers launched may be less.

Next, there are two small unstabilized satellites under development. One is for the study of the ionosphere and the other for solar physics and cosmic rays. The two satellites will be launched as co-operative projects between NASA and ESRO. NASA intends to provide a Scout launcher which can put a payload of $75 \mathrm{~kg}$ into a circular orbit of about $500 \mathrm{~km}$ altitude or, in the case of the ionospheric satellite, into an eccentric orbit of $350 \mathrm{~km}$ perigee and $1200 \mathrm{~km}$ apogee.

Scientific proposals have indicated a strong desire for satellites with some form of stable orientation, in particular for astrophysical studies. Stabilized satellites of this kind would be medium in size and would be capable of being launched by a Thor-Delta vehicle. The development of one such satellite has been agreed upon. It would have a weight of about $200 \mathrm{~kg}$ and would be placed into an orbit of $500 \mathrm{~km}$ altitude for the purposes of stellar astronomy.

Finally, it has been agreed to develop and build a large astronomical satellite (LAS) the details of which will be given under topic $(4 a)$ of this session.

The time schedule of the agreed initial program is about 3 years for the small unstabilized satellites (1967), 4 years for the small stabilized satellite ( 1968$)$ and 6 years for the large astronomical satellite (1970).

Further plans, which have not yet been approved in detail, are the launching of six small 
unstabilized satellites over the period of the next 8 years, and six or eight medium-sized stabilized satellites. In addition, seven space probes or satellites with highly eccentric orbits are planned during the 8 year period. One such vehicle would be ready for the time of the next solar maximum and one a year later.

Finally, another large project is being considered, starting in I966. Alternative proposals for this second large project are being assessed. They include a lunar mission, with soft landing of instruments or a lunar satellite, and a fly-by mission to a comet.

\section{The Sun}

\section{(a) C. de fager. The ESRO Solar Program}

The ESRO solar program includes, for $1_{96} 6_{5}$, the launching of an unstabilized rocket for the measurement of the solar X-ray flux between $3 \AA$ and $65 \AA$, and for 1966 , the launching of an unstabilized satellite (ESRO II) for the measurement of solar soft X-rays, $\mathrm{He} 30_{4} \AA$, and Lyman-alpha, and for cosmic ray experiments. It is hoped that experiments with stabilized rockets and satellites will take place at a later time, but no final decisions have as yet been taken.

\section{(b) H. F. Smith. NASA Plans for Solar Astronomy}

The United States National Aeronautics and Space Administration program in solar physics is carried out with the aid of aircraft, balloons, sounding rockets, Earth satellites and deep space probes. Major efforts are being devoted to the current series of Orbiting Solar Observatories (OSO) and to the incipient series of Advanced OSOs.

OSO is a standardized 500-pound spacecraft designed to accommodate interchangeable experiments for the purpose of solar research. It consists of two major sections, the wheel and the sail. The wheel rotates at $30 \mathrm{rpm}$ to provide inertial stabilization, keeping its plane within a degree of the Sun's direction. The experiments are accommodated in five wheel compartments. The sail counterrotates to remain normal to the Sun's direction during the daylight phase. An experiment compartment in the sail either points at the Sun with a precision of a minute of arc, or else performs a 40 line raster scan in a four minute period. Planned orbits will be circular at a height of 300 nautical miles, and inclined 33 degrees to the equator.

A total of eight OSO missions are contemplated. The first, OSO-1, was launched in 1962. Subsequent launches, starting late in 1964 , will occur at 9 to 12 month intervals. Experiments assigned to the first five OSO missions are enumerated in the following table:

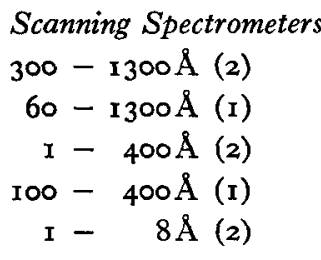

\section{Photometers}

Helium $304 \AA$ ( I)

Lyman-alpha (2)

Soft X-rays: $\lambda>2 \AA(4)$

$$
\lambda<2 \AA \text { (2) }
$$

Hard X-rays (10-100 keV) (3)

Low Energy $\gamma$-rays $(<100 \mathrm{MeV})(8)$

High Energy $\gamma$-rays $(>100 \mathrm{MeV})(5)$

\section{Spectroheliographs}

Lyman-alpha (2)

2- $20 \AA$ (3)

White Light Coronagraph (1)

Miscellaneous

Neutrons (I)

Earth Albedo (I)

Emissivity (3)

Meteoroid-detectors (I)

Zodiacal Light (2)

UV Sky Spectrometer (I)

Night Sky Glow (I) 
The next list gives the OSO-E experiments:

(a) Wheel Compartments

I. Hydrogen Lyman-alpha absorption photometer. J. Blamont, Univ. Paris.

2. Low energy solar gamma-ray studies, $5 \mathrm{keV}$ to $\mathrm{I} 50 \mathrm{keV}$ by scintillation detector. K. Frost, Goddard SFC. (OSO-B follow-on.)

3. Broad-band solar spectrophotometry, $760 \AA$ to $103 \circ \AA$. W. Rense, Univ. Colorado.

4. Solar X-ray monitoring by ion chambers, $0^{\circ} \AA$ to $16 \AA$. T. Chubb, Naval Res. Lab. (OSO-B follow-on.)

5. Zodiacal light monitor at 90 degrees from the Sun, by filters and photocells. E. Ney, Univ. Minnesota (OSO-B follow-on.)

(b) Pointing Compartment

6. X-ray spectroheliograph, using proportional counters in $8-18 \AA$ and $3_{-9} \AA$ bands. E. Stewardson, Univ. Leicester and R. Boyd, Univ. College, London.

7. Scanning spectrometers $1-400 \AA$ J. Lindsay, Goddard SFC. (OSO-I and OSO-C follow-on.)

8. Lyman-alpha spectroheliograph, J. Purcell, Naval. Res. Lab. (OSO-B follow-on.)

The second major undertaking is the Advanced Orbiting Solar Observatory (AOSO). This spacecraft and a typical set of experiments are currently in the design and development phase. Its objectives are functional versatility, accurate pointing, and the ability to accommodate experimental instruments up to 2.5 meters in length. AOSO will point with a precision of 5 seconds of arc anywhere within a 40-minute square centered on the Sun. It will also scan over the entire 40 -minute square (in a I20-line raster over a 25 -minute period) or any 5 -minute square within it (in a 60 line raster over a 5 -minute period). The planned 300 nautical mile circular polar retrograde orbit will permit nearly continuous sunlit operation over several months. Up to $25^{\circ}$ pounds of payload weight can be accommodated in the pointing compartment which measures 40 inches in diameter by 100 inches in length.

The instruments selected for prototype development are listed below. Final decisions about the flight plan have not been made, but the first launch will occur no sooner than 1968 . Tentatively the initial schedule shows four AOSO vehicles launched at 6 month intervals.

AOSO-Experiments :

I. Nine-inch telescope and scanning spectrometer, 300-1300 $\AA$. Goldberg, Parkinson and Reeves, Harvard College Observatory.

2. White light coronagraph. Eddy and Newkirk, High Altitude Observatory.

3. Five seconds of arc resolution X-ray telescope. Lindsay and Giacconi, Goddard Space Flight Center.

4. Lyman-alpha and helium line spectroheliographs. Purcell and Tousey, Naval Res. Lab.

\section{Discussion}

$R$. N. Thomas asked whether any satellite observations were planned to achieve spectral resolution of 0.05 to $0.02 \AA$ anywhere in the region between 200 and $\mathrm{r} 200 \AA$. He considers this to be very critical for theoretical investigations of $(a)$ theory of spectral line formation in the chromosphere and corona, and $(b)$ details of the structure in the transition region chromospherecorona. Dr Smith answered that, thus far, no proposal for an experiment with such high spectral resolution had been received. The President pointed out that, at this stage of development, the experiments were aimed at high angular rather than high spectral resolution. To L. Biermann's question concerning the highest angular resolution possible at present, $D r$ Smith answered that the pointing accuracy is now $I$ minute of arc but will reach 5 to $I$ second of arc in the near 
future. $R$. Lüst remarked that only experiments measuring electromagnetic radiation of the Sun were reported. He wonders which vehicles will carry instruments to measure solar particle streams. Dr Smith explained that the orbit of the OSOs was not adequate for such experiments. However, the Explorer vehicles will carry flare-detection systems and instruments measuring soft X-ray and Gamma-ray fluxes. F.-C. Pecker enquired whether it would be possible to obtain simultaneous spectroheliograms in Lyman-alpha and in the $\lambda 2800 \mathrm{Mg}$ II lines. Such observations could be very interesting for theoretical interpretations, inasmuch as the line formation is quite different for the two lines. Since such observations are technically very difficult to make, $H$. F. Smith suggested the use of the Ca II lines for comparison instead. With reference to the suggested observations of the $\lambda 2800 \mathrm{Mg}$ II lines, Y. Ohman mentioned that he had such a project in the ESRO program. He also has started a joint effort with J. Lindsay at the Goddard Space Flight Center, where preparations are at present being made by Miss Kerstin Fredga and others for the launching of two rockets in the spring of 1965 . The rockets will be equipped with birefringent filters which are being made for Prof. Öhman by Kovo on the Solc principle. If successful results are obtained, the problem of taking simultaneous photographs in various wavelengths will be considered, particularly during flare events. In reply to Mrs Herzberg's question, $\mathrm{Dr}$ Smith said that the time resolution depends on the scanning rate of the instrumentation and that it could reach a fraction of a second. Furthermore, Mrs Herzberg asked whether simultaneous observations will be obtained of the $\lambda_{304} \mathrm{He}$ line and of hard X-rays. Dr Smith hopes to correlate these observations within a second or so in OSO-D.

\section{(c) V.K. Prokofiev. On The Far UV Spectrum Of The Sun}

In the following two communications, Prof. Prokofiev reported on some work concerning the far UV spectrum of the Sun which has been carried out in the U.S.S.R. The first is a paper by S. L. Mandelshtam, S. P. Fedoseev, E. Y. Cononov, and S. V. Lebedev on the interpolation of the far UV solar spectrum. These authors have carried out laboratory experiments at the Physical Institute of the Academy of Sciences of the U.S.S.R. in order to interpret the solar spectrum in the wavelength region between $15^{\circ}$ and $300 \AA$. Using a vacuum spark between electrodes of pure iron, they obtained a series of spectra which they suggest are due to iron ions in various stages of ionization, probably Fe v to Fe IX. Many of the lines in these spectra coincide with lines in Tousey's rocket UV spectra of the Sun. The wavelength measurements are accurate to about $\pm 0.04 \AA$.

Prof. Prokofiev's second communication was a paper by S. L. Mandelshtam, I. A. Shitnik, V. V. Crutov, and L. P. Malyavkin dealing with photographs of the Sun in the far UV. A rocket was launched on 1963 June 6 in order to take pictures of the Sun in various wavelength bands of the region $\lambda 200-400 \AA$. The rocket reached an altitude of $500 \mathrm{~km}$. The useful duration of the flight within the altitude range $120 \mathrm{~km}$ to $500 \mathrm{~km}$ was 570 seconds. The equipment consisted of 12 pinhole cameras, the diameters of the holes ranging from 0.14 to $0.28 \mathrm{~mm}$. The holes were covered with organic and $\mathrm{Al}$ films of different thicknesses $(0 \cdot 2 \mu-5 \mu)$, which permitted the observations in the wavelength bands $10-100 \AA, 170-400 \AA$ and near $800 \AA$. The exposure times varied from ro to $\mathbf{5 7 0}$ seconds. One block of cameras was oriented towards the Sun in two axes. The pointing accuracy in one of the axes was about $\pm 2^{\prime}$. The orientation of the second axis was relatively ineffective and resulted in motion of the image with an amplitude of $40^{\prime}$. The authors compared the photograph of the Sun thus obtained in the wavelength band $170-400 \AA$ with a picture made by Tousey on 1963 May 5 i.e. 27 days earlier, and showed that the centers of activity are essentially unchanged after one solar rotation.

No discussion followed these two papers except for a question by the President who asked Prof. Prokofiev whether any plans existed for continuous monitoring of the Sun in the $304 \AA$ He line. Prof. Prokofiev answered in the affirmative. 


\section{Moon, Planets, Comets, and Interplanetary Medium}

\section{(a) L. Biermann. ESRO Projects}

The following experiments (part of which are still in the planning stage) were briefly reported.

I. Rocket release experiments.

$\mathrm{R}$ I2. Spectroscopic study of gas clouds (e.g. $\mathrm{NH}_{3}$ ) released above $100 \mathrm{~km}$ (Liège).

$\mathrm{R}$ 33. Ion cloud ( $\mathrm{Ba}$ II, $\mathrm{Sr} \mathrm{II}$ ) above $I 50 \mathrm{~km}$ released as $\mathrm{Ba} I$ and $\mathrm{Sr} I$ with subsequent photoionization (Munich).

2. Spectroscopic studies with stabilized rockets.

Plans aiming at such studies of the Moon, of planets and of comets are being undertaken in London, Liège, and Munich.

3. Satellite experiments.

$\mathrm{S}$ I6. Ion cloud in interplanetary space (Munich, like $\mathrm{R} 33$ ).

$S$ r. Measurement of the electron density in interplanetary space (Munich).

$\mathrm{S}_{24}$ and S 35. Measurement of magnetic fields and energetic particles in interplanetary space (British group).

$\mathrm{S} 5^{8}$ and $\mathrm{S} 73$. Investigation of the properties of the solar wind (Bruxelles-Utrecht, Bari-Roma).

4. First ESRO satellite in a highly eccentric orbit.

One payload will combine several cosmic ray experiments with measurements of the plasma and magnetic fields in interplanetary space, the priority probably being given to plasma experiments.

5. Second large ESRO project (SLEP).

The feasability of a cometary mission is being studied at the present time.

\section{Discussion}

In the discussion that followed this report, Mrs Heraberg asked whether CO was being considered in connection with the gas cloud releases. Prof. Biermann explained that the release of $\mathrm{CO}$ in interplanetary space would involve too many complications. F. Zwicky commented that he had long advocated the firing of narrow jets of gases (e.g. Li, Be, B) from shaped charges or other launchers. The source of the resulting light would be far more concentrated and the particles would cause a number of chain reactions. To this comment, Dr Lüst replied that such experiments would be reasonable if one were interested in the Earth's atmosphere. However, for the investigation of interplanetary space, one tries to go beyond $300 \mathrm{~km}$ just to avoid the chain reactions that are produced by the Earth's atmosphere at lower altitudes. Prof. Swings pointed out that the Liège experiment was primarily planned to obtain cometary information and not to study the Earth's atmosphere. For this reason the instrumentation must be launched to as high an altitude as possible.

\section{(b) U. Liddel. NASA Projects}

Two additional Ranger flights are scheduled for the calendar year 1965. They are not expected to exceed the performance of Ranger VII, but may hopefully equal it. It is planned to photograph other maria such as Tranquilitatis, which are of interest to the Apollo program.

Three Surveyor test flights (soft-landed spacecraft) are scheduled for 1965 , but these are not expected to provide any scientific data. Television pictures and studies of the physical nature of the lunar surface will be made in operational Surveyor flights scheduled for the calendar year 1966 . The area that can be observed by television may be severely limited by the small radius of the Moon. However, much should be learned about the nature of the surface. Equipment will be included to give some idea of the Moon's chemical composition from the back 
scattering of alpha particles (Chicago). Seismic activity will be studied with a single axis seismometer (Calif. Inst. Tech., and Columbia Univ.).

The next major advance in the astronomical study of the Moon will be made by the three Lunar Orbiters scheduled for the calendar year 1966. The precise orbits to be used have not yet been determined, but they could vary from equatorial to polar. The area of interest to the Apollo program is $\pm 10^{\circ}$ in latitude and $\pm 60^{\circ}$ in longitude. Within this area, the Orbiter will have the capability of photographing up to $4 \times 10^{4}$ square kilometers with a resolution of 8 meters. Also, it will be able to cover an area up to 8000 square kilometers with a resolution of I meter. Lastly, it will have the capacity to cover some 200 square kilometers sufficiently well to detect a landed Surveyor spacecraft.

Pictures will be recorded on film for later transmission to Earth by command. Since the Orbiter must 'see' both the Sun and the Earth while transmitting pictures, the transmission time is limited to approximately 45 minutes per orbit. The period of the Orbiter is approximately two and a half hours.

The phase angle between the line of sight of the camera and the Sun line is $60^{\circ}$. The angle between the line of sight and the normal to the surface affects the quality of the pictures; for example, it has been calculated that the intensity of light will drop to half-value for $15^{\circ}$ variation away from the Sun. The angle of view of the low resolution camera (about $20^{\circ}$ ) will cause some variation in light intensity across the frame for even a flat surface. However, topographical features can still be recognized.

In addition to photography (and perhaps after all the pictures have been taken) precise orbital measurements will be made which will permit gravitational field measurements of the Moon. Plans have also been made to study the micro-meteorite and high-energy particle fluxes. Following the original series of Orbiters, other measurements such as thermal mapping radar reflection, magnetic measurements and other experiments will be considered.

Two Mariner spacecraft are scheduled to be launched towards Mars in the last quarter of this year. The prime astronomical interest here is the capability of the television system to provide 20 pictures of the planet, the nearest approach distance being approximately $18000 \mathrm{~km}$ from the center of the planet. Red and green filters will be used alternately in taking the exposures, which will be recorded on magnetic tape and transmitted to Earth at the rate of one per day. The pictures will be composed of an array of $200 \times 200$ points with 64 shades of gray. The design resolution is $5 \mathrm{~km}$. Because we are in such great need of information concerning the atmospheric density of the planet, the spacecraft trajectory is so designed that telemetric signals will be occulted by the planet for perhaps a period of $\mathbf{r}$ hour. This requires a passage of the planet from rather near the south pole across the Southern Hemisphere towards the equator.

The Mariner spacecraft will record and transmit various data concerning the interplanetary medium such as cosmic radiation, magnetic fields, solar plasma, etc. Although the trajectory near the planet is at the distance required by biological considerations to prevent accidental contamination, it is hoped that some indication may be obtained of the magnetic field, ionized particle concentration, and micro-meteorite density of the Martian surroundings.

Investigations of the interplanetary field by two flights of Pioneer spacecraft are planned in 1966. These will include studies of the solar plasma, cosmic radiation, and magnetc fields.

\section{Discussion}

$P$. Swings inquired whether NASA had any plans for studying comets. $U$. Liddel answered that plans for cometary experiments over the next Io years were still only on paper. $F$. Zwicky proposed the following experiment for determining the water content of the rocks on the Moon. When approaching the Moon, the Rangers might fire projectiles (for instance from shaped 
charges) towards the Moon, with the impact as seen from the rocket being televised back to Earth. Projectiles made of $\mathrm{Al}$ or other reducing elements, upon penetrating the Moon's surface, would hydrolize any crystal water that might exist in the rocks. If present, the crystal water in hydrolizing would produce $\mathrm{H}_{2}$ at great pressures which in turn would expel material from the Moon. This material being hot would radiate and reveal its existence in the spectrum of the Balmer emission lines.

\section{(c) V.K. Prokofiev. Reflection of Laser Beam from the Moon}

The 2.6 meter telescope at the Crimean Astrophysical Observatory was used in conjunction with a ruby laser to send a light beam to the Moon and to receive its reflection from the lunar surface. The experiment was carried out on $\mathrm{I} 693$ September 13 from $\mathrm{I}^{\mathrm{h}} \circ 0^{\mathrm{m}}$ to $2^{\mathrm{h}} 3^{\mathrm{m}}$ U.T. The diameter of the beam was I I mm with a divergence angle $\alpha=3$ ", which was reduced to $\circ^{\prime \prime} 5$ after collimation by the telescope. The pulse duration was 2 microseconds. Owing to atmospheric scattering, the divergence angle of the incoming reflected signal was $8^{\prime \prime}$. Thus the diameter of the lunar area covered by the laser beam was $14 \mathrm{~km}$. A cooled $\left(-70^{\circ} \mathrm{C}\right)$ photomultiplier was employed to receive the reflected beam. Thirty pulses were directed to the crater Albategny. The number of photoelectrons per impulse was $n_{b}+n_{s}=6 \cdot 2$. The background, $n_{b}$, was measured upon receipt of the reflected signal first during 3 microseconds, then during 10 seconds. Its average value was $n_{b}=4.67 \pm 0.38$. From this, the value of the signal $n_{B}=\mathrm{I} \cdot 53$ was determined. Since it is four times greater than the standard deviation of the $n_{b}$ measurements, it appears that the reflection of the laser beam from the Moon's surface could be reliably registered.

\section{Stars and Stellar Systems}

\section{(a) R. Lüst. The ESRO Large Astronomical Satellite (LAS)}

From the very beginning of the planning of ESRO it has been emphasized that one of the major projects of this organization should be a Large Astronomical Satellite (LAS). These plans have been discussed at some length, and a number of different design studies carried out. The main discussions have taken place in a small working group chaired by Dr Butler while the design studies have been undertaken in three different member countries.

Although the detailed scientific requirements are still under consideration, it is possible to summarize some of the tentative conclusions that have emerged from the preliminary discussions and design studies. The main objective is to obtain UV spectra of stars in the spectral region between 900 and $3000 \AA$, with a resolution of $\mathrm{I} \AA$ or better. In addition, a secondary program for the observation of X-rays will very probably be included.

With respect to the design of the satellite, the following points are of particular relevance: (I) Consideration must be given to the fact that the first ultraviolet sky scans will be comlpeted before the ESRO satellite is ready for launching. (2) The planned LAS should not be just a single project, but rather the culmination of an extensive development program which should include special experiments with sounding rockets and smaller satellites. A program of laboratory experiments and tests on materials such as UV mirror coatings, UV intensity standards, etc., should also be included.

The size, weight, and shape of the satellite will be largely determined by the choice of a launching vehicle. The new European rocket launcher, which is now being built by another European organization, the ELDO, will probably be used. The first stage will be the Blue Streak rocket, the second, either the Scout or a modified version of the French Veronique, while the third stage is being developed in Germany.

It has been stated that the vehicle will be capable of launching about $1000 \mathrm{~kg}$ into a circular orbit of $500 \mathrm{~km}$ altitude. The launching will take place from Woomera (Australia). A near polar 
retrograde orbit of $98^{\circ}$ has been discussed whereby the orbital plane rotates with the same angular velocity as the apparent motion of the Sun around the Earth, thus keeping the SunEarth direction in the plane of the orbit, at all times.

The dimensions of the payload for the ELDO launcher are as follows: Cylinder with a diameter of $\mathrm{I} \cdot 9 \mathrm{~m}$, topped by a cone, the total length being $4 \mathrm{~m}$ and the length of the cylindrical part being $2.5 \mathrm{~m}$. The specifications for this satellite are given below in two parts. The first part concerns the basic vehicle, and the second the specific scientific instruments and the scientific objectives.

I. The basic vehicle:

(a) It is felt that the vehicle should contain a platform which can be pointed in any direction with an accuracy of $I$ minute of arc in sunlight as well as in shadow.

(b) The space available for scientific instrumentation should have a total length of not more than $2 \mathrm{~m}$ and be able to house a mirror of not more than $\mathrm{I} \mathrm{m}$ diameter.

(c) The weight available for scientific instrumentation should be about $200-300 \mathrm{~kg}$.

2. For the scientific instrumentation itself, the following specifications are under discussion:

(a) The primary instrument will be expected to make stellar and if possible nebular spectrophotometric observations extending from $3300 \AA$ to about $1100 \AA$ and if possible down to 9 I $2 \AA$.

(b) Given the magnitude of the stars which it is hoped may be observed, the primary mirror should exceed $50 \mathrm{~cm}$ in diameter, and may reach $70-80 \mathrm{~cm}$. But the possibility of two parallel mirror systems is also under consideration, since this might be the most efficient way to observe the longer and the shorter UV at the same time.

(c) High spectral resolution is required throughout the whole spectrum. It is felt that a few tenths of an angstrom is a reasonable target.

(d) A secondary instrument for observing X-rays should be smaller than and subordinate to the first one and be mounted parallel to the optical telescope. The broad-band wavelength region has not yet been chosen.

(e) If possible some other secondary experiments might be included.

3. The satellite is to consist of two main sections:

(a) A reference system, such as a platform whose position is fixed in space;

(b) a movable part which can be pointed in any direction.

Two preliminary design studies have been proposed, their difference lying in the choice of the relative weights for the two main sections. For the OAO, more or less the whole vehicle is pointed at the object to be observed, while the fixed reference system to which the vehicle is connected weighs relatively little. The other proposed design is one that has a very heavy platform fixed in space to which a movable telescope is attached.

No decisions have been made so far on these technical details. It is hoped that the actual work of building this satellite will start within a few months and that it will be completed in 1970.

\section{(b) P. Swings. ESRO Projects}

With respect to the stars and stellar systems, the European astronomers are mainly interested in the LAS of ESRO which has been described by R. Lüst. On the other hand, many European projects of experiments with rockets depend on the availability of stabilized rockets. These should be soon at our disposal. Certain rocket experiments will be done as preparation for the LAS and for the small stabilized satellites. 
I. Rocket experiments. They concern mainly:

(a) the far UV spectroscopy or photometry or spectrophotometry of stars (Butler, Boyd, Heddle, etc.), or of the integrated night sky (Butler);

(b) the measurement of stellar and galactic soft X-ray radiation (de Jager, Underhill, van Gils); the search for discrete X-ray sources with high sensitivity low noise X-ray detectors (Stewardson).

2. Satellite experiments.

The solar experiments with the unstabilized ESRO II satellite have been described by C. de Jager.

The experiments within the ESRO ad hoc group on Stars and Stellar Systems require a stabilized satellite. They are concerned with:

(a) scanning of the sky in the far UV (Liège-Edinburgh: Butler, Houziaux, Monfils);

(b) scanning of the sky in the infra-red (Delbouille);

(c) stellar spectrography in the UV in a small region with $2 \AA$ resolution (Underhill and de Jager);

(d) possibly scanning of the sky in the region of soft X-rays (de Jager).

Other stellar experiments, such as the variability of non-solar discrete X-ray sources (Stewardson) and the search for stellar X-rays (Boyd and Stewardson) will probably be secondary experiments aboard the LAS.

Projects which have been submitted but have not yet been discussed by the ESRO ad hoc working group on Stars and Stellar Systems are not included in this report.

\section{Discussion}

Miss Roman asked for the wavelength range under consideration for scanning the sky in the infra-red. L. D. Delbouille replied that single bands will be used in the range $I^{\cdot} 2$ to $3^{\circ} \circ \mu$, or I. 8 to $3 \cdot \circ \mu$, and that they might use even larger bands.

\section{(c) Nancy Roman. NASA Projects}

The following projects were briefly outlined. More detailed information can be found in the NASA 'Blue Book' on Opportunities for Participation in Space Flight Investigations, June I964, Washington, D.C. 20546.

I. Small vehicles.

(a) Balloons, reaching altitudes of about $30 \mathrm{~km}$ :

Project Stratoscope.

Gamma-ray observations.

(b) Research Airplanes:

The X-I 5 airplane can reach altitudes of about $100 \mathrm{~km}$ and may stay at that altitude for some 5 to 8 minutes. Payloads of about 90 pounds can be carried. The pointing capability is $I^{\prime}$. (Photography of Orion Nebula.)

(c) Rockets:

Aerobees, reaching altitudes of $160-300 \mathrm{~km}$, with a net payload of $100-300$ pounds. Journeyman, reaching altitudes of $1500-2900 \mathrm{~km}$ with a net payload of $5^{\circ}-\mathrm{I} 75$ pounds.

2. Explorers.

(a) Geodetic Beacon Explorer:

$\begin{array}{llll}\text { Ready for: } & \text { 3rd quarter I964 } & 3^{\text {rd quarter I965 }} & \text { 2nd or } 3 \text { rd quarter I966 } \\ \text { Launch angle: } & 80^{\circ} & 50^{\circ} & \\ \text { Altitude: } & 1000 \pm 200 \mathrm{~km} ; & \text { I 100 to } 3000 \mathrm{~km} & 1000 \mathrm{~km}\end{array}$


Equipment: 162 and $324 \mathrm{MHz}$ transmitter; flashing light: 10-20 sets of five flashes each separated by $4^{\mathrm{s}} ; 5^{\circ}$ field predictions good to $1^{\circ}$ one week in advance; optical corner reflector.

(b) Geodetic Balloon Explorer:

Ready for the first half of 1966 ; launch angle about $90^{\circ}$; altitude about $3000 \mathrm{~km}$.

3. Observatories.

(a) Orbiting Solar Observatory (OSO).

OSO-B2: Hallam, I500-3000 $\AA, \lambda / \Delta \lambda \sim$ 10, $1.5-6.0$ magnitude B-stars. Frost, $0 \cdot 1-3 \mathrm{MeV}$ Gamma-rays. Ney, Zodiacal Light.

OSO-C: Peterson, $15 \mathrm{keV}-600 \mathrm{keV}$ Gamma-rays. Kraushaar, $\sim$ Ioo MeV Gamma-rays.

OSO-D: NRL, Lyman-alpha night sky glow ion chambers.

OSO-E: Frost, 5-I $50 \mathrm{MeV}$ Gamma-rays. Blamont, Lyman-alpha profile and flux. Ney, Zodiacal Light.

(b) Orbiting Geophysical Observatory (OGO).

OGO-A and B: Haddock, solar bursts and galactic background, 2-4 $\mathrm{MHz}$. Mange, Geocoronal Lyman-alpha ion chamber to monitor interplanetary Lyman-alpha. Wolff, Gegenschein photometry in UV, green and infra-red regions.

OGO-C and D: Haddock, galactic mapping at 2.5 and $3.0 \mathrm{MHz}$.

OGO-E: radio-astronomy, Gamma-ray spark chambers.

(c) Orbiting Astronomical Observatories (OAO).

OAO-A: Smithsonian Astrophysical Observatory: Project Celescope, $\lambda$ 2600, 2300, I 500, I $400 \AA$; I2-inch telescopes, observation of about 50000 stars brighter than $8 m^{m}$; exposure times about 60 seconds.

Wisconsin: four stellar photometers, 8-inch telescopes, band pass of about $300 \AA$, in the wavelength region $\lambda$ II 50 to $4200 \AA$. 16-inch nebular photometer, in the wavelength region $\lambda 2000-3300 \AA$.

Two $7 \times$ 12-inch objective gratings, 20 or $200 \AA$ slit, wavelength range $\lambda 2000-4000 \AA$.

OAO-B: 36 -inch objective grating, 10 or $100 \AA$ slit, wavelength region $\lambda$ 1000$2000 \AA$, and $1050-4000 \AA$ at $2,8,64 \AA$ resolution.

OAO-C: 32 -inch objective grating, wavelength region $\lambda 700-4000 \AA, 0.1$ and $0.4 \AA$ slits, for stars brighter than $6^{m}$.

Henize: UV sky photographs.

Univ. of London: $\mathrm{X}$-ray telescope with three wavelength ranges between 2 and $100 \AA$.

Future plans: Radio astronomy Explorer; X-ray astronomy Explorer.

The National Aeronautics and Space Administration invites the scientific community to participate in these near-future flight missions with any scientific project for the investigation of space.

\section{Discussion}

$P$. Swings expressed the gratitude of the world-wide scientific community to NASA for its generosity in inviting guest scientists to participate in the planned space flight missions. A. Maxwell stressed the desirability for an astronomical satellite with a large radio antenna to be carried up to altitudes of about $600 \mathrm{~km}$, and he wondered whether NASA already had such a project. Miss Roman explained that no such satellite had as yet been formally scheduled 
because of financial difficulties and lack of man-power. F. G. Smith mentioned plans for a satellite-borne radio telescope, possibly to be placed in ESRO II, for studyingthe galactic radio emission at $\mathrm{I} \mathrm{MHz}$. Special attention will be given to a high angular resolution.

\section{(d) V.K. Prokofiev. Satellite Tracking}

Prof. Prokofiev reported on two different methods of satellite tracking which have recently been employed in the U.S.S.R. The first was used by P. P. Dobronravin for the observation of distant space probes, such as Mars I and Lunik IV. These space probes were photographed with the $2.6 \mathrm{~m}$ Shajn telescope of the Crimean Astrophysical Observatory. An image intensifier was attached to the prime focus of the telescope, making it possible to obtain good images of space probes up to around ${ }_{1} 3^{\text {th }}$ to $I_{5}$ th magnitude with $I$ to 3 seconds exposure time. The beginning and the end of each exposure was registered with an accuracy of 0.01 second. By measuring the position of the space probe relative to the stars on the photographic plate, it is possible to determine the coordinates of space probes with an accuracy of 5 seconds of arc.

The second method is an application of a sensitive TV system to observe artificial satellites. It was employed by E. S. Agapov, V. F. Anisimov, V. M. Mozherin, V. B. Nikonov, V. V. Prokofyeva, V. I. Pergament, and S. M. Sinenok. They showed that modern TV systems could increase sensitivity by more than a factor of roo compared to ordinary photography. TV systems make it possible to observe and register the position of artificial satellites without any difficulty. With a lens of only $80 \mathrm{~mm}$ diameter, satellites could be observed down to 9 th magnitude. For regular satellite tracking the TV systems can be extremely useful.

\section{SHORT COMMUNICATIONS}

\section{On Space Observations of Cool Stars and Planets}

Y. Fujita outlined several observations of cool stars and planets which should be made from above the Earth's atmosphere.

(a) Infra-red observations of cool stars. It is desirable to make photometric observations of M-type stars in the spectral region $\mathrm{I} \cdot 6$ to $\mathrm{I} \cdot 8 \mu$ where the emergent flux is expected to reach its maximum value. Observations so far have shown that the energy distribution of the emergent flux of these stars is approximately black body radiation. This should be checked from above the Earth's atmosphere. Concerning Mira type variables, the amplitude of the light curve in the infra-red is considered to be small, but this has not yet been ascertained. Infra-red observations should be made to clarify this point, which is important for the pulsating theory by F. Kamijo (Publ. astr. Soc. Japan, 15, I, 1963). There are other types of variable stars which should be observed above the Earth's atmosphere in the infra-red. These are the $\mathrm{R}$ Corona Borealis type variables. According to the ideas of F. Kamijo (Publ. astr. Soc. Fapan, 15, 440, 1963) their light variation should be increased on account of graphite particles produced by gases ejected from the star. If solid particles absorb only the visible light, then, as the temperature rises, heat must be emitted in the infra-red. This means that the light curve may show little or no variation in the infra-red.

(b) Ultra-violet observations of cool stars. Observations in the ultra-violet region seem very difficult but they are very desirable for studying the dynamical situation of cool stars. Some investigators believe that the atmospheres of the red supergiant stars are excited by the ultraviolet radiation of the central star. Observations in the Lyman-alpha region could verify this idea. Furthermore, it is also believed that the emission lines of Miratype variables originate from atoms which are excited by Lyman-alpha radiation from convective elements, something like the spicules of the Sun. Such convective elements may be revealed by observation in the 
extreme ultra-violet emission lines, i.e. in the wavelength region shorter than $\lambda$ rooo $\AA$. Finally, observations in the X-ray region of flare stars of main sequence M-type stars may clarify the flare phenomena.

(c) Infra-red observations of planets. In the photographic region up to about $\mathrm{I} \cdot 2 \mu$ it would be desirable to investigate the existence of $\mathrm{O}_{2}$ and $\mathrm{H}_{2}$ which are masked by the Earth's atmosphere. With high dispersion spectrographs it might be possible to draw maps of the wind velocity distribution on Mars, since the fine motion of the atmosphere can be measured. On the other hand, in the far infra-red region it might be possible to detect spectral lines of some molecules which are not abundant on planets. This, of course, assumes that detectors for the region of fundamental vibrational bands of molecules are available. These bands can be very useful in determinations of the abundance of molecules.

\section{Space Observations of Eclipses}

f. Houtgast stressed the importance of observing total solar eclipses from rockets carrying the instruments outside or nearly outside the Earth's atmosphere. Such observations will eliminate the various disturbances due to the Earth's atmosphere and, in addition, by adequate launching of the rocket can prolong the duration of the eclipse. This is especially important for the critical eclipse phases of second and third contact. On second contact the intensity drops at the Sun's limb by a factor of 10000 to 100000 ; for a stationary observer, this occurs in less than half a second. A rocket moving along the Moon's shadow could extend this time by a factor of 100. A few rocket observations during solar eclipses have already been made, i.e. by Tousey for X-rays and by Ney for coronal structures and intensities. Dr Houtgast expressed the hope for close collaboration between the Solar Eclipse Working Group of Commission I2 and this Commission.

\section{Extraterrestrial Studies of the Solar Corona}

G. Newkirk described some plans for extraterrestrial studies of the solar corona. The High Altitude Observatory hopes to make the following three related investigations of the solar corona outside of eclipse above the atmosphere:

(a) In the spring of 1965 , there are two flights planned for the Coronascope II system, which successfully observed the corona at distances from $I \cdot 6$ to 5.25 solar radii on 1964 March 5 . These flights will be timed in order to compare previous observations with coronal observations obtained during the eclipse of I 65 May 30.

(b) A similar coronagraph is planned for the AOSO satellite. The image will be telemetered to the ground at a rate of one picture every 90 minutes or, during special periods of interest, a rapid scan of one picture every 3 to 5 minutes. Since the lifetime of the satellite is expected to be about 1 year from the launch date ( 1968 ), this coronagraph will allow the examination of the evolution and rotation of coronal streamers during the early maximum stages of the next solar cycle. It should be mentioned that a similar coronagraph has been built by the Naval Research group under the direction of Dr Tousey for flight in the OSO-B which is scheduled to be launched this winter.

(c) There are plans for a program of several balloon-borne coronagraph flights to supplement observations made with the AOSO instrument. Since this balloon-borne instrument will be capable of higher angular (ro seconds of arc) and time (several pictures per minute) resolution than will the orbital coronagraph, it will be flown during a particularly intense period of solar activity to study transients, which presumably occur in the corona during such events as solar flares. 


\section{Transition Layer and Chromosphere of the Sun}

$Z$. Suemoto made some remarks concerning the transition layer and the chromosphere of the Sun.

(a) Transition layer chromosphere-corona. It would be very useful to compare the line intensities in the extreme ultra-violet with the radio intensities in the decimeter and centimeter regions. The extreme UV radiation of medium excitation yields the abundances of the elements other than hydrogen whereas radio emission gives the abundance of hydrogen. Comparisons of this kind may explain the anomalous chemical composition in the transition layer and its heterogeneous structure. However, solar radio radiations, including the thermal component, are subject to large changes due to solar activity. Hence, it is very likely that radio intensities of relatively high frequencies, supposed to originate primarily in the transition layer, are affected by active centers extending into the corona. On the other hand UV observations appear to indicate that lines originating in the transition layer do not change appreciably in intensity with solar activity. Therefore, in order to compare the extreme UV line emission with radio emission, observations should be made during the time of sunspot minimum. Such experiments are being carried out at present but should be repeated during a period of sunspot minimum. In connection with the heterogeneous model proposed by Moriyama and Suemoto, a one dimensional scan across the disk in one of the extreme UV lines may give some idea as to whether the transition layer is highly heterogeneous or not.

(b) The chromosphere. Observations in a radial direction involve the difficult problem of separating the relatively weak chromospheric emission component from the bright Fraunhofer spectrum of the photosphere. This applies even to the case of the strong $\mathrm{H}$ and $\mathrm{K}$ lines of $\mathrm{Ca}$ II. The situation can be improved when observing the UV Mg II lines, since their intensities are much stronger than the $\mathrm{Ca}$ II line intensities relative to the underlying Fraunhofer spectrum. Accurate line profile measurements of the $\mathrm{Mg}$ in lines are very desirable observed in undisturbed and in active regions. With good spatial resolution, high resolution spectrograms and high quality spectroheliograms in $\mathrm{Mg}$ II would yield better quantitative information of the detailed structure in the chromosphere than so far has been obtained from the Ca II lines. Similarly, Lyman-alpha line profiles can be valuable on account of the absence of underlying photospheric lines. However, in this case hydrogen absorption in the neighborhood of the Earth complicates the line profile studies.

\section{Discussion}

The President pointed out that the most recent observations of the solar $\mathrm{Mg}$ II lines by Dr Tousey were made with very high spectral resolution and, therefore, are expected to represent the true, or nearly the true, profiles of these lines.

\section{X-ray Images of the Sun}

G. Elwert presented a new device for forming X-ray images of the Sun which was developed at Prof. Mollerstedt's Institute in Tübingen. The method is to reduce the usual zone plates electron optically. An electron image is produced on a silver layer. This silver layer then is placed in an electrolytic bath and a copper layer grows on all parts where no electrons have struck the silver surface. This copper pattern can be pulled off. With the usual zone plates, the radius of the outermost ring is a few millimeters and that of the innermost ring is some tenths of a millimeter. The focal length being given by $f=r^{2} / \lambda$, where $r$ is the radius of the innermost circle, the resulting focal length is about ro $\mathrm{m}$ for soft $\mathrm{X}$-rays at about $\lambda=5 \circ \AA$. If, however, one reduces the zone plate electron-optically by a factor of Io, the focal length of the reduced zone plate becomes of the order of $10 \mathrm{~cm}$ at $\lambda=50 \AA$. The resolution of the device is given by $\alpha=\mathrm{I} \cdot 2(\lambda / D)$, where $D$ is the diameter of the plate. For the reduced zone plate and wavelength o 
of $5 \circ \AA$, the diameter $D$ is of the order of some seconds of arc. On the other hand, the resolution of a pin-hole camera with a pin-hole diameter $d$ and a camera length $l$ is given by $d / l$. With $d$ equal to the diameter of the outermost ring of the reduced zone plate and with a camera length of about $20 \mathrm{~cm}$, one obtains a resolution of several minutes of arc corresponding to one-tenth of the solar diameter. The X-ray pictures of the Sun taken by Friedman had such a resolution. For comparison Dr Elwert showed two pictures of wire grids of different sizes, one taken with a pin-hole camera, the other using a reduced zone plate. In both cases the $\mathrm{X}$-ray source was the $\mathrm{K}_{a}$ radiation of $\mathrm{Al}$ at $8 \cdot 4 \AA$, the distance to the source was $\mathrm{I} \mathrm{m}$, and the image distance was I.75 m. The pin-hole camera had a pin-hole diameter of $200 \mu$, and could show the $600 \mu$ wire grid. The zone plate had a diameter of $280 \mu$, so that nearly the same number of quanta passed through the pin-hole and the zone plate. The focal length of the zone plate was $63 \mathrm{~cm}$ for a wavelength of $8{ }_{4} \AA$; at $\lambda 25$ it would be about $20 \mathrm{~cm}$. The theoretical resolution was about I second of arc. The zone plate picture reproduced the image of the $600 \mu$ wire grid, an $80 \mu$ wire grid (the distance between the neighboring grids seen under an angle of $\mathrm{I}_{4}$ seconds of arc), and a very thin wire grid of $8 \mu$ diameter seen under an angle of about I second of arc. This showed that the theoretical resolution was nearly attained.

\section{Discussion}

In reply to $H$. $E$. Hinteregger's question, $D r$ Elwert said that the exposure times were approximately the same in both the pin-hole and the zone plate pictures.

\section{Determination of Time on the Moon's Surface}

$N$. Boneff briefly mentioned a method for determining the terrestrial universal time on the Moon's surface, assuming that the telecommunication with the Earth were interrupted. The method was first proposed by him two years ago ( 1962 ) at the XIIIth Astronautical Meeting held in Bulgaria. The method consists of observing stellar occultations by the Earth, which is always in the zenith of an observer at the center of the Moon's disk. The problem is to devise the best instrument fitted to lunar conditions.

The President thanked all the contributors to the scientific session of the Commission and adjourned the meeting. 